\title{
Characterization of placenta-derived mesenchymal stem cells cultured in autologous human cord blood serum
}

\author{
LIBIN WANG $^{1,2^{*}}$, YINXUE YANG ${ }^{1 *}$, YONGZHAO ZHU $^{1}$, XIAONA MA ${ }^{1}$, TING LIU ${ }^{1}$, GUANGYI ZHANG $^{1}$, \\ HENG FAN $^{1}$, LIJUN MA ${ }^{1}$, YIRAN JIN ${ }^{1}$, XIURUI YAN ${ }^{1}$, JUN WEI $^{1,2}$ and YUKUI LI ${ }^{1}$ \\ ${ }^{1}$ Ningxia Human Stem Cell Institute, the Affiliated Hospital of Ningxia Medical University; \\ ${ }^{2}$ Key Laboratory of Fertility Preservation and Maintenance of Ningxia Medical University, Yinchuan, Ningxia, P.R. China
}

Received March 1, 2012; Accepted July 12, 2012

DOI: $10.3892 / \mathrm{mmr} .2012 .1000$

\begin{abstract}
Human placenta-derived mesenchymal stem cells (P-MSCs) have drawn increasing attention in the field of stem cell research due to their potential in clinical applications as well as their rich and easy to procure cell source. While studies demonstrating the potential of P-MSCs for therapeutic transplantations have been documented, a clinically compliant procedure for P-MSC expansion in vitro has yet to be established. To this end, previous studies have demonstrated that MSCs of bone marrow and cord blood origins cultured in human cord blood serum (hCBS) are comparable to those cultured in fetal bovine serum (FBS), indicating that hCBS may be an alternative to FBS for the development of in vitro cell expansion procedures free of animal components. However, stem cells from origins other than bone marrow or cord blood, particularly from human placental tissues, which have demonstrated a good potential for clinical applications, have not been characterized under similar conditions. In this study, in an attempt to define a clinically compliant protocol for P-MSC expansion in vitro, we examined the effects of human hCBS as a replacement for FBS on cell proliferation capacity, differentiation potential, MSC-specific phenotypic expression and the genetic stability of P-MSCs in cultures. P-MSCs expanded in vitro in autologous hCBS maintained the capacity of self-renewal and expressed surface antigens
\end{abstract}

Correspondence to: Dr Jun Wei and Dr Yukui Li, Ningxia Human Stem Cell Institute, The Affiliated Hospital of Ningxia Medical University, Yinchuan, Ningxia 750004, P.R. China

E-mail: lydiajunwei@hotmail.com

E-mail:yukuili@hotmail.com

*Contributed equally

Abbreviations: P-MSCs, human placenta-derived mesenchymal stem cells; hCBS, human cord blood serum; DP, discrimination power; NSE, neuron-specific enolase; GFAP, glial fibrillary acidic protein; GAP-43, growth-associated protein-43; NF-M, neurofilament; SCGE, single cell gel electrophoresis

Key words: cord blood serum, stemness, placenta-derived, mesenchymal stem cell characteristic of bone marrow-derived mesenchymal stem cells. Under differentiation conditions, the P-MSCs expanded in hCBS developed into adipogenic, osteogenic and neurogenic cell phenotypes. Chromosomal karyotyping and single cell gel electrophoresis analysis demonstrated that P-MSCs cultured in autologous hCBS were genetically stable. These results suggest that autologous hCBS may be used as an alternative to FBS for the in vitro expansion of P-MSCs for clinical applications.

\section{Introduction}

Mesenchymal stem cells (MSCs) are currently being increasingly investigated for their therapeutic potential in regenerative medicine and inflammatory conditions (1-4), due mainly to their multi-lineage differentiation potential (5) and their limited expression of MHC class II antigen $(6,7)$, as well as their immunomodulatory functions (8-11). The former allows MSCs to be applied to a wide range of clinical conditions, while the latter allows MSCs to be used not only for autologous but also for allogeneic transplantations. These application potentials strongly suggest a need for identifying a stem cell source that is capable of providing plentiful and ethically acceptable yet easily procured stem cells. MSCs are traditionally identified and isolated from bone marrow and bone marrow-derived MSCs (BM-MSCs) remain the most commonly investigated MSCs (2). Previous studies have shown some limitations of BM-MSCs to their wide range of application, including that they have to be isolated through an invasive procedure, that they exist in bone marrow in a very low ratio (12), and that their differentiation capacity decreases as the donor's age increases (13). In recent years, increasing attention has been placed on placenta-derived MSCs (P-MSCs) $(6,14)$. Besides sharing major properties with BM-MSCs (15), P-MSCs have shown certain unique properties that make them a prime candidate for stem cell-based therapies. Such properties include: i) Placental tissues originate from the early stage of embryonic development, which may logically lead to the hypothesis that MSCs from these tissues may have retained the plasticity of their mother stem cells (14). This hypothesis has been supported by recent studies showing that MSCs from amniotic and chorionic membranes are pluripotent and may differentiate towards not only a mesodermal lineage but also into ectodermal $(16,17)$ and endodermal (18) cells. ii) Placenta is the tissue that maintains fetomaternal 
immune tolerance during pregnancy, indicating that cells from this tissue may have immunomodulatory functions. This is evidenced by the observation that P-MSCs in the fetomaternal interface show higher immunosuppressive effects than adult MSCs (19). iii) P-MSCs can be procured from term placentas in large supply, free of invasive procedures and ethical complications. A recent scale-up culture study showed that a range of 7.6-11.6x $10^{8}$ of passage 2 P-MSCs can be obtained from approximately half of a placental tissue mass, and such cells maintain a 0.6-0.8/day doubling rate through passage 5 (20), indicative of placenta as a unique and plentiful source of MSCs for clinical applications.

Of equal importance to identifying proper stem cell source for cell-based therapies is the establishment of clinic-compliant methods for in vitro cell expansion, which should result in cells free of safety complications, yet maintain full stemness for expanded cells. Previously, the majority of expansion protocols for scale-up production of MSCs used medium supplemented with fetal bovine serum (FBS) (21). It has been observed that patients transplanted with T cells grown in FBS supplemented medium developed IgG immunity against FBS proteins (22), and that xenogenic proteins retained in MSC cytoplasm may have elicited immunological responses in vivo following cell transplantation (23). The concerns of xenogenic protein effects have drawn interest in using serum-free conditions. However, the long-term effects of serum-free medium have yet to be defined, since cells cultivated in serum-free conditions have demonstrated contradictory results (21). To this end, the replacement of FBS with human serum has been explored. While adult human serum has been found to present several significant limitations (24), autologous human cord blood serum (hCBS) and autologous adult human serum have been successfully tested for the in vitro expansion of hematopoietic stem cells (25), T cells (26), as well as BM-MSCs and cord blood-derived MSCs $(27,28)$.

Furthermore, the amniotic side and maternal side of the placenta may isolate fetal and maternal origin P-MSCs; therefore, it is crucial to verify the origins of P-MSCs. Owing to short tandem repeats (STRs) having polymorphisms and high mutation rates, they are broadly used for cell authentication. STRs are short tandemly repeated DNA sequences that involve a repetitive unit of 1-6 bp. Strand-slippage replication gives rise to the mutation of STRs and to different individual DNA samples containing the length-variant STR alleles, which may be separated by polyacrylamide gel electrophoresis.

Placental tissues are developmentally different from other MSC origins, and P-MSCs are developed in vivo in an environment different from other MSCs. In an attempt to establish a clinically compliant protocol for P-MSC processing, the characteristics of P-MSCs cultured in autologous hCBS were investigated as a replacement for FBS. In this study, we demonstrate that P-MSCs expanded in autologous hCBS maintain their self-proliferation capacity and multi-lineage differentiation potential, express a MSC-specific phenotype, and are genetically stable.

\section{Materials and methods}

Isolation and culture of P-MSCs. Full-term placentas were obtained from healthy mothers at the time of routine elective caesarean section in the Affiliated Hospital of Ningxia Medical University. Informed consent was obtained from each mother prior to delivery. The placental tissues were immerged under aseptic conditions into protection solution containing $98 \%$ DMEM-low glucose (DMEM-LG; Invitrogen, Carlsbad, CA, USA), $1 \%$ hCBS (prepared in the laboratory) and $1 \%$ penicillin/streptomycin solution (Sigma, St. Louis, MO, USA), and transferred to the laboratory within $30 \mathrm{~min}$ for processing. Pieces of placental tissue approximately 1-cm-thick were dissected from the amniotic side of the placentas, and cut into small pieces approximately $1 \mathrm{~mm}^{3}$ in size, washed in PBS (Invitrogen) to remove blood cells, and then digested with a combination of $270 \mathrm{U} / \mathrm{ml}$ collagenase IV (Roche, Penzberg, Germany) and $240 \mathrm{U} / \mathrm{ml}$ dispase II (Roche) at $37^{\circ} \mathrm{C}$ for $1 \mathrm{~h}$ in a shaker water bath. After digestion, the cells in suspension were filtered through $150 \mu \mathrm{m}$ filters (BD, Franklin Lakes, NJ, USA), collected by centrifugation at $100 \mathrm{x}$ g for $5 \mathrm{~min}$, washed 3 times, and seeded in 24-cm flasks.

Three different media were used for comparison. Medium I: DMEM-LG supplemented with 10\% FBS (Invitrogen), 2\% L-glutamine and $1 \%$ penicillin/streptomycin solution; medium II: Same as medium I with the exception that the FBS was replaced by $10 \%$ autologous hCBS; medium III: Same as medium I with the exception that the FBS was replaced by $10 \%$ non-autologous hCBS. Cells in all media were cultured in a $37^{\circ} \mathrm{C}, 5 \% \mathrm{CO}_{2}$, humidified incubator. Upon reaching $80 \%$ confluence, cells were harvested with $0.25 \%$ trypsin/0.05\% EDTA (Invitrogen) and subcultured by a 1:3 dilution. The cell doubling cycle was determined by dividing the cell number increase during a given passage with the length of time (hour) of that passage. After passage 3, cell samples from each culture were cryopreserved in solution containing 60\% DMEM-LG, 30\% hCBS and 10\% dimethyl sulphoxide (DMSO, Invitrogen).

To verify the fetal origin of P-MSCs, maternal P-MSCs were isolated from tissues $0.5-\mathrm{cm}$-thick from the maternal side of the same placentas.

Verification of the fetal origin of P-MSCs. Blood samples were collected from parents of the newborn babies whose placentas had been used for isolation of fetal and maternal P-MSCs. Informed consent was obtained from the parents prior to sample collection. DNA was prepared from the two types of P-MSCs as well as the from parental blood samples using an AxyPrep kit (Axygen). Four known STR polymorphic sites $(29,30)$ were selected for genetic matching. Polymerase chain reaction (PCR) primers for these STR sites are shown in Table I. PCR was carried out using an EasyTaq PCR SuperMix kit (Transgen, Beijing, China) for 30 cycles under the following conditions: $94^{\circ} \mathrm{Cx} 50 \mathrm{sec}$, annealing temperature x50 sec, $72^{\circ} \mathrm{Cx} 40 \mathrm{sec}$. PCR results were viewed by polyacrylamide gel electrophoresis.

Phenotypic characterization of P-MSCs by flow cytometry. All monoclonal antibodies used for flow cytometry were obtained from BD Pharmingen (Franklin Lakes, NJ, USA), and prepared in PBS. Single cell suspensions were made from cell cultures by trysinization and prepared in PBS. Cells $\left(1 \times 10^{6}\right)$ were incubated in $150 \mu \mathrm{l}$ with one of the following antibodies for $10 \mathrm{~min}$ at room temperature: IgG1-PE, CD29-PE, 
Table I. PCR primers for polymorphic tandem repeat markers and expected discrimination powers.

\begin{tabular}{|c|c|c|c|c|c|}
\hline Gene site & Primer sequence & $\operatorname{Tm}\left({ }^{\circ} \mathrm{C}\right)$ & $\begin{array}{l}\text { Polymorphic } \\
\text { fragment (bp) }\end{array}$ & $\begin{array}{l}\text { Discrimination } \\
\text { power (DP) }\end{array}$ & Combined DP \\
\hline D2S1399 & $\begin{array}{l}\text { 5'-CATTGGTCCAGGTAAACTGC-3' } \\
\text { 5'-TTCACAAGGTTCCACAAGGT-3' }\end{array}$ & 55 & $136-176$ & 0.958 & \multirow{4}{*}{0.9999926} \\
\hline D10S2325 & $\begin{array}{l}\text { 5'-CTCACGAAAGAAGCCTTCTG-3' } \\
\text { 5'-GAGCTGAGAGATCACGCACT-3' }\end{array}$ & 58 & $208-233$ & 0.948 & \\
\hline GATA198B05 & $\begin{array}{l}\text { 5'-GTGGAGCCAATTCACCTAAT-3' } \\
\text { 5'-GAGAGACAGAACCTATAGCATGC-3' }\end{array}$ & 55 & $112-152$ & 0.9533 & \\
\hline D18S535 & $\begin{array}{l}\text { 5'-TCATGTGACAAAAGCCACAC-3' } \\
\text { 5'-AGACAGAAATATAGATGAGAATGCA-3' }\end{array}$ & 55 & $131-163$ & 0.927 & \\
\hline
\end{tabular}

CD34-PE, CD73-PE, IgG2a-FITC, CD14-FITC, CD44-FITC, CD45-FITC, CD90-FITC, CD105-FITC or HLA-DR-FITC. After this incubation, cells were resuspended in $300 \mu \mathrm{l}$ PBS. Flow cytometry analysis was performed on a Calibur cytometer (BD Biosciences).

Multi-lineage differentiation of P-MSCs. Differentiation of P-MSCs towards adipogenic, osteogenic and neurogenic lineages was performed in vitro. P-MSCs at passage 3 were used for all differentiation studies. Adipogenic differentiation was induced by culturing $70 \%$ confluent P-MSCs for 10 days in DMEM-LG medium supplemented with 10\% FBS, $1 \mu \mathrm{M}$ dexamethasone, 1mM 3-isobutyl-1-methyl-xanthine, $10 \mu \mathrm{g} / \mathrm{ml}$ insulin and $60 \mu \mathrm{M}$ Indole Miyuki-3-isobutyl-1-methylxanthine (Sigma). Adipogenic differentiation was assessed by oil Red O staining. Osteogenic differentiation was induced by culturing $70 \%$ confluent P-MSCs for 14 days in DMEM-LG containing $10 \% \mathrm{FBS}, 100 \mathrm{nM}$ dexamethasone, $10 \mathrm{mM} \beta$-glycerophosphate (Sigma), $0.05 \mathrm{mM}$ ascorbic acid-2-phosphate (Wako Pure Chemical Industries, Tokyo, Japan) and $2 \mathrm{mM}$ L-glutamine. Osteogenic differentiation was assessed by alizarin red staining. Neurogenic differentiation was induced by culturing $50 \%$ confluent P-MSCs for 12 days in DMEM-LG containing 10\% FBS, 2 mM L-glutamine, 2\% DMSO, $200 \mu \mathrm{M}$ butylated hydroxyanisole (BHA, Sigma) and $25 \mathrm{ng} / \mathrm{ml}$ NGF. Neurogenic differentiation was assessed by immunostaining for the neural cell-specific proteins neuron-specific enolase (NSE), glial fibrillary acidic protein (GFAP), neurofilament (NF-M), growth-associated protein-43 (GAP-43) and nestin.

Karyotyping and single cell gel electrophoresis. P-MSCs from passages 2, 14 and 18, and from cryopreserved passage 6 samples were karyotyped by Giemsa staining of metaphase chromosomes and analyzed on a Cytovision analyzer (Applied Imaging, Wetzlar, Germany). DNA stability of P-MSCs was assessed for passage 2 cells by single cell gel electrophoresis (SCGE) using an OxiSelect Comet Assay kit (Cell Biolabs, San Diego, CA, USA) according to the manufacturer's instructions. For DNA damage quantification, 50 cells in the SCGE plate of each sample were randomly selected under a microscope, and diameters of nuclei (comet head) and tail moment (tail length) were measured. DNA damage was represented by a parameter of tail moment/head ratio.

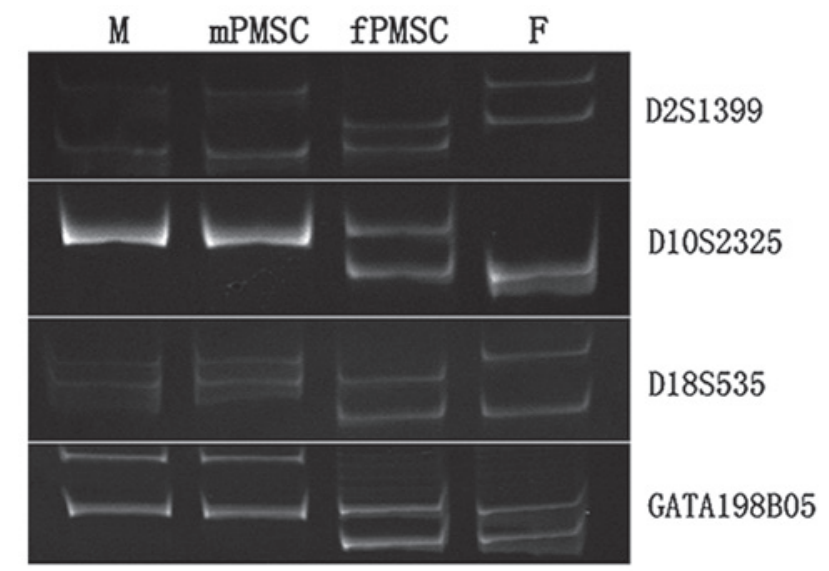

Figure 1.Genotype verification of polymorphic tandem repeat loci. Polymorphic repeat loci were PCR-amplified and separated by electrophoresis in polyacrylamide gels. Each column represents 4 polymorphic markers PCR-amplified from DNA templates of different origins. M, mother blood sample; mPMSC, P-MSC sample isolated from mother side of a placenta; fPMSC, P-MSC sample isolated from fetal side of a placenta; F, father blood sample.

Statistical analysis. Data are expressed as the means \pm standard error and analyzed by the student's-test. A value of $\mathrm{P}<0.01$ was considered to indicate a statistically significant difference.

\section{Results}

Verification of P-MSCs for their fetal origin. Placental tissues contain P-MSCs of both fetal and maternal origin, originating respectively from the amniotic and maternal side of the placenta. To verify that the P-MSCs isolated from the amniotic side were of fetal origin, 4 polymorphic STR markers were used for parent-offspring genetic matching (Table I). These polymorphic markers individually identify the genetic origin with higher than $90 \%$ discrimination power (DP), and when combined, they give higher than $99.999 \%$ DP. As shown in Fig. 1, for all the markers tested, the P-MSCs from the amniotic side of the placenta carry alleles of maternal and paternal origin, indicative of the fetal genome, while the P-MSCs from the maternal side of the placenta carry alleles of only maternal origin. This test was performed for multiple samples with 

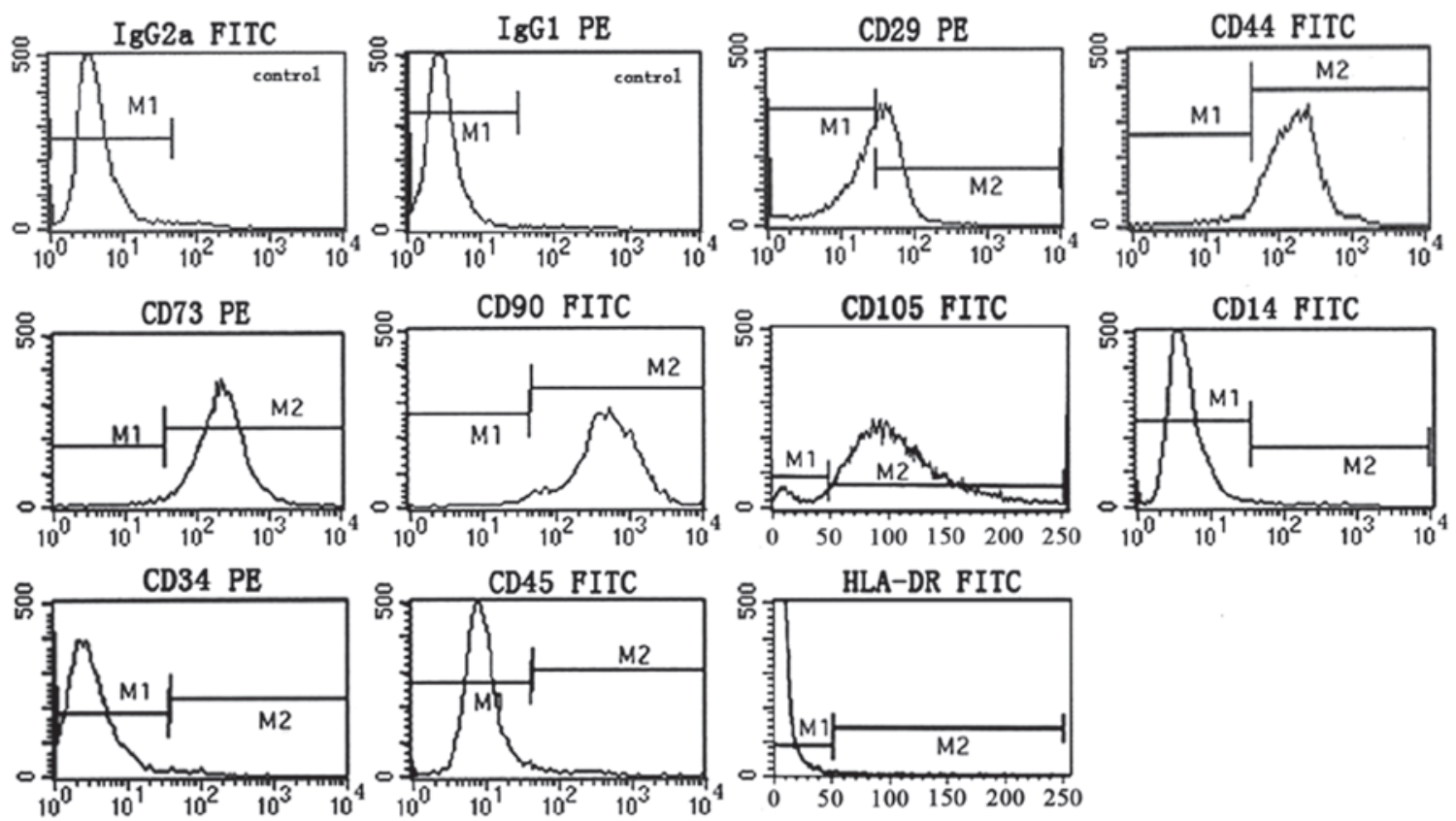

Figure 2. Flow cytometry analysis for the expression of cell surface antigens by P-MSCs grown in autologous hCBS. IgGaFITC and IgG1PE are isotope controls for the antibody types used in flow cytometry. P-MSCs, human placenta-derived mesenchymal stem cells; hCBS, human cord blood serum.

consistent results. Therefore, 1 -cm-thick tissue from the amniotic side of placentas was used for all fetal P-MSC isolations.

P-MSCs growing in hCBS maintained the MSC phenotype. It is generally accepted that MSCs are positive for surface antigens CD73, CD90 and CD105, and negative for CD34, CD45 and CD14, with restricted expression of HLA-DR (31). Flow cytometric phenotype analysis of passage 3 P-MSCs grown in hCBS showed that the cells are positive for CD29, CD44, CD73, CD90 and CD105, and negative for CD14, CD34, CD45 and HLA-DR, indicative of a typical MSC phenotype (Fig. 2). These results demonstrate that hCBS supported the expression of a normal MSC phenotype.

P-MSCs growing in hCBS maintained multi-lineage differentiation potential. MSCs are characterized by the potential of differentiation towards mesenchymal lineages (31). Recent studies also showed that P-MSCs may contain pluripotent cells that are capable of differentiating towards ectoderm and endoderm lineages (16-18). To test whether hCBS supports P-MSC differentiation potential, we performed adipogenic and osteogenic (mesenchymal lineage, Fig. 3G and H, respectively), as well as neurogenic (ectodermal lineage, Fig. 3A-E), differentiation of P-MSCs. Differentiation protocols for all the three lineages have been previously used for stem cell differentiations (see Materials and methods). The results indicate that P-MSCs grown in hCBS also differentiated into the ectodermal lineage.

P-MSCs growing in hCBS are genetically stable. Genetic stability is one of the most important characteristics of cultured cells when their clinical application is concerned, and is affected by cell culture conditions. To verify that hCBS are capable of supporting clinically compliant in vitro expansion of P-MSCs, we tested the genetic stability of P-MSCs cultured in hCBS at a cellular level by chromosome karyotyping and at the DNA level by SCGE. Karyotyping for P-MSCs at passages 2, 14 and 18 in cultures, and from cryopreserved cells of passage 6, revealed no detectable genetic variation (Fig. 4). SCGE analysis showed normal nucleus movement for hCBS cultured P-MSCs, either prior to or following cryopreservation, and no difference in nucleus movement between cells cultured in hCBS and in FBS (Fig. 5).

\section{Discussion}

In this study, we demonstrate that homologous hCBS may be used as a replacement for FBS in the in vitro expansion of human P-MSCs. Both cell proliferation capacity and cell differentiation potential, two basic properties of stem cells, were well maintained in P-MSCs when they were cultured in sustained passages as well as when recultured following cryopreservation. P-MSCs growing in hCBS were genetically stable and showed no difference in DNA damage tests when compared with cells growing in FBS. These results may serve as a starting point for the development of a clinically compliant procedure for in vitro P-MSC expansion, an alternative to FBS-based or serum-free protocols.

MSCs have drawn increasing interest for their therapeutic potentials, and hence their cell sources are becoming an issue of concern. It has been estimated that one MSC may be found in every 10,000 nucleated cells in the bone marrow of a human newborn, and this ratio would decrease by 10 -fold in the marrow of the teenage population, and by 100 -fold in the marrow of the elderly population (32). By contrast, human placentas have proven to be a rich and easy to procure MSC source. In this study, it was observed that, when cultured in hCBS, P-MSCs showed a similar phenotype and multiple 


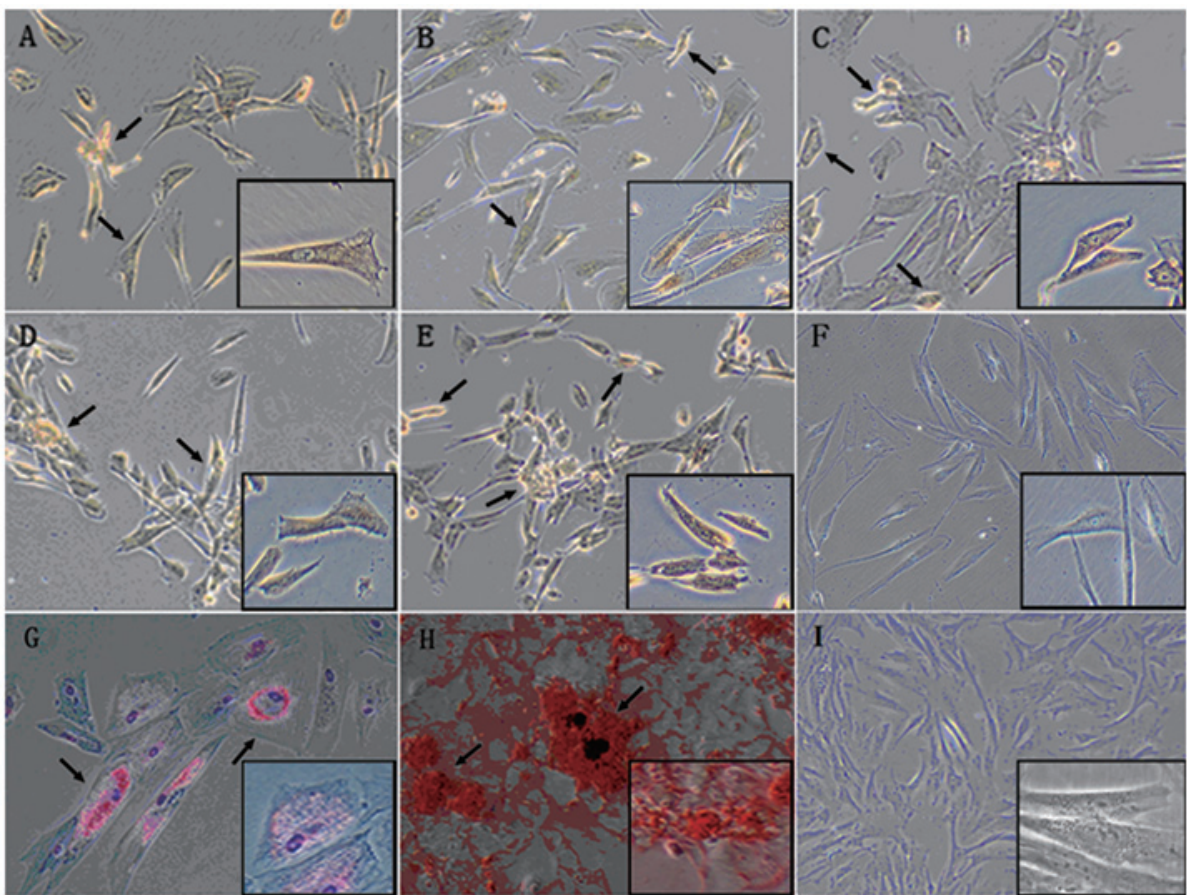

Figure 3. Differentiation of P-MSCs cultured in hCBS towards adipogenic, osteogenic and neurogenic lineages (x200 and x400). Neural cells characterized by immunohistochemistry staining for (A) neuron specific enolase (NSE), (B) glial fibrillary acidic protein (GFAP), (C) nestin, (D) growth associated protein-43 (GAP-43), (E) neurofilament medium polypeptide (NF-M), and (F) negative control, respectively. (G) Adipocyte-like cells characterized by oil Red O staining. (H) Osteocyte-like cells characterized by Alizarin Red S staining. (I) Negative control. P-MSCs, human placenta-derived mesenchymal stem cells; hCBS, human cord blood serum.
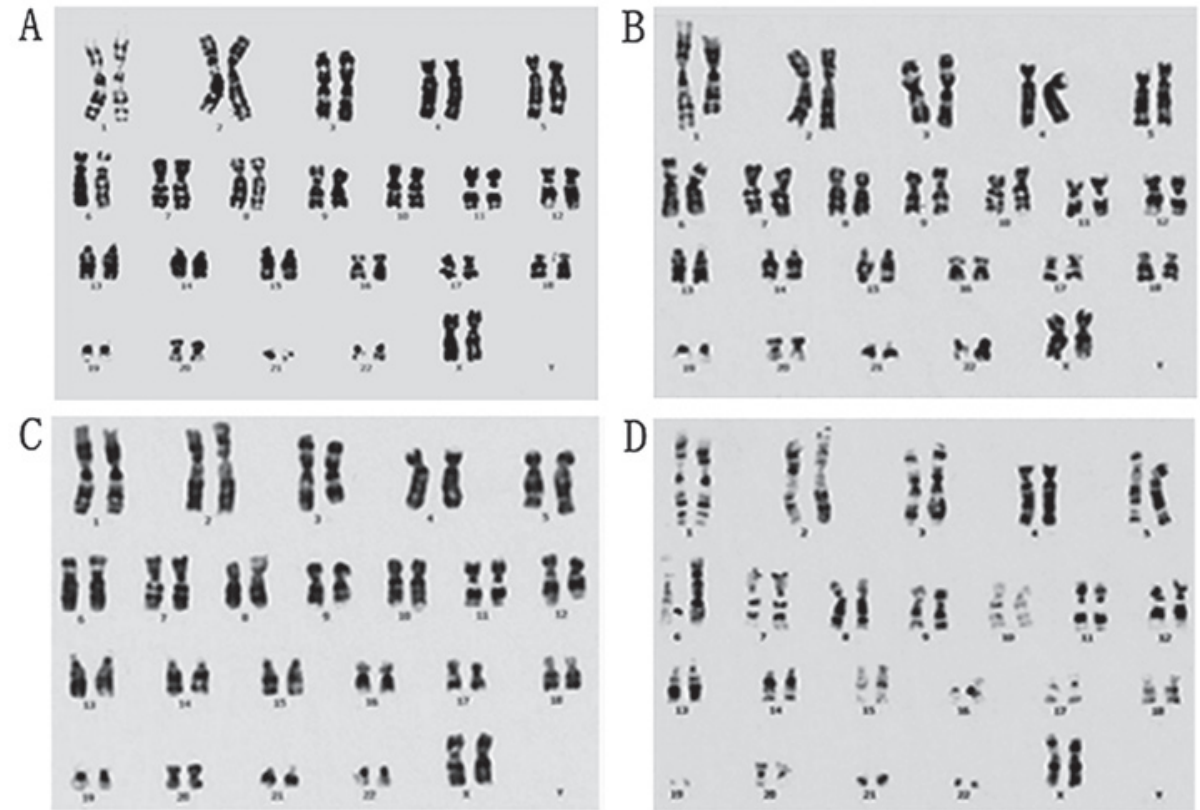

Figure 4. Chromosome karyotypes of P-MSCs cultured in hCBS (x200). (A, B and C) Cells of passages 2, 14 and 18 in culture, respectively, and (D) cells of passage 6 from cryopreservation were analyzed. Karyotyping was performed by Giemsa staining of metaphase chromosomes and analyzed on an automated analyzer (see Materials and methods). Each karyotype represents a total of 30 metaphases analyzed for each group. Karyotype normality was determined by clinical diagnostic standards. P-MSCs, human placenta-derived mesenchymal stem cells; hCBS, human cord blood serum.

differentiation potentials in comparison with BM-MSCs, as characterized by cell surface antigen typing and as tested for the generation of mesenchymal and ectodermal cell lineages, respectively. These observations are in agreement with certain earlier studies using various culture systems (31).
Recently, a study showed that P-MSCs, when compared with BM-MSCs, appeared to be more immunogenic and to have less immunomodulatory functions (33). In this study and previous studies (31), a significant immunogenic phenotype of P-MSCs was not observed. The different observations in 

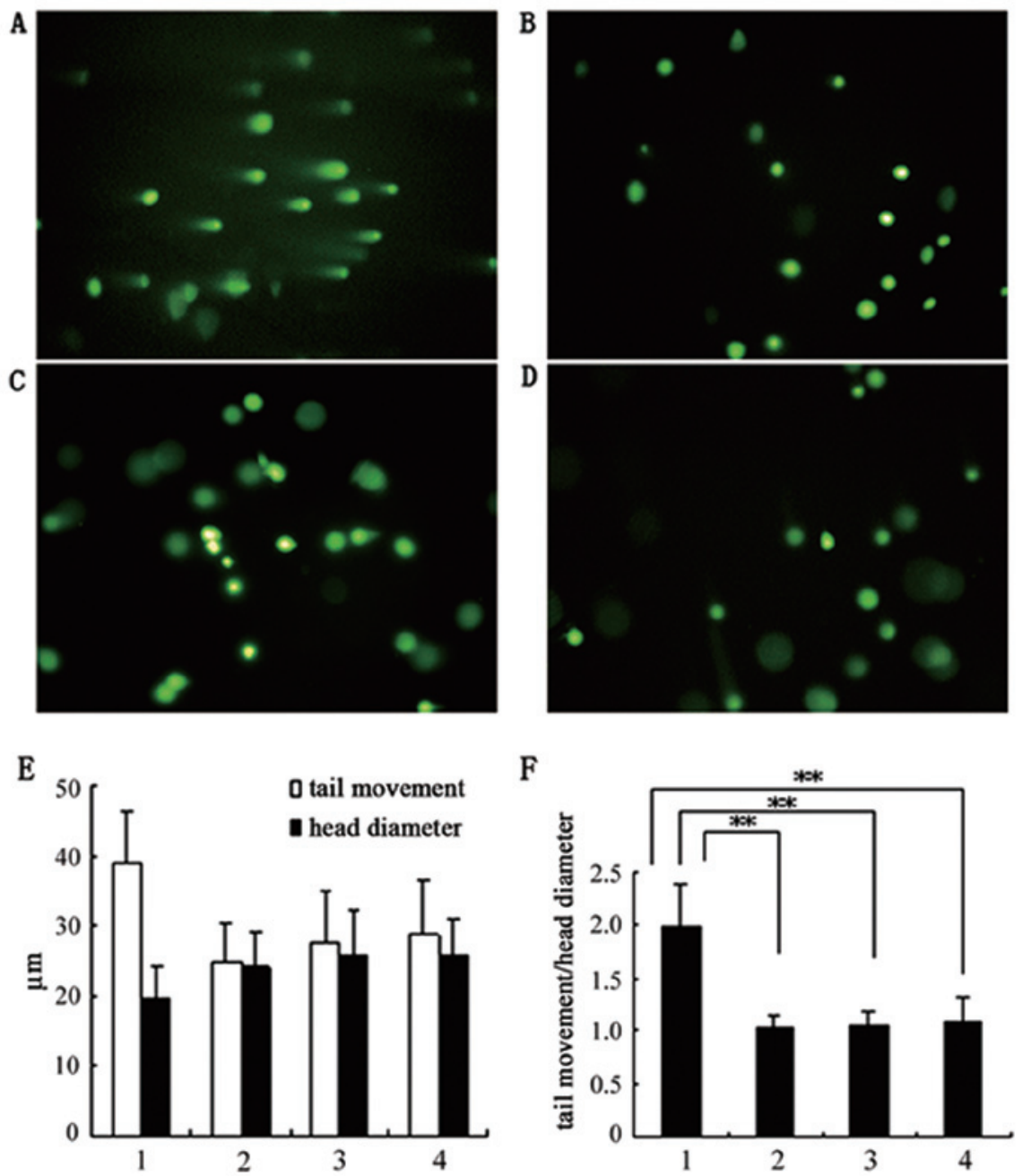

Figure 5. DNA damage analysis by single cell gel electrophoresis (SCGE) (x200). Passage 2 P-MSCs cultured in hCBS and FBS were analyzed and quantified. (A) Control. P-MSCs treated with $5 \% \mathrm{H}_{2} \mathrm{O}_{2}$ for 15 min prior to electrophoresis showed DNA damage as detected by comet tail moments. No DNA damage was detected in the SCGE assay for P-MSCs either in hCBS (B) prior to and (C) following cryopreservation, or (D) in FBS. (E and F) DNA damage quantification as measured by comet tail moment. (E) Measurements of nucleus diameters and tail moment length. (F) Nucleus moment index as presented by ratios of nucleus diameters against tail moments ${ }^{(*} \mathrm{P}<0.01$, group 1 vs. group 2,3,4). (E and F) 1, P-MSCs in FBS treated with $\mathrm{H}_{2} \mathrm{O}_{2}$; 2 , $\mathrm{P}-\mathrm{MSCs}$ in $\mathrm{FBS}$; 3 , $\mathrm{P}-\mathrm{MSC}$ in hCBS prior to cryopreservation; 4, P-MSCs in hCBS following cryopreservation. P-MSCs, human placenta-derived mesenchymal stem cells; hCBS, human cord blood serum; FBS, fetal bovine serum.

different studies may be partly due to the difference in cell populations tested or in the cell culture conditions employed. In Fazekasova et al's study (33) a mixture of fetal and maternal P-MSCs were tested. Although the immunogenic properties of maternal P-MSCs are less understood, it may be different from that of fetal P-MSCs, as shown in a previous study where P-MSCs of fetal origin (from amniotic membranes) revealed higher immunomodulatory functions than BM-MSCs (19). In our study, we isolated fetal P-MSCs and verified their genetic origin. These fetal P-MSCs showed no significant HLA-DR expression when cultured in hCBS, in agreement with earlier studies with amniotic membrane cells. The fetal identity of P-MSCs may be advantageous when clinical application is concerned, since a homologous genetic background of cells may reduce possible complications. At the same time, the isolation of fetal P-MSCs excluded a possibility that the growth of fetal P-MSCs may be partially supported by maternal cells in the same culture, hence leading to the conclusion that hCBS may provide sufficient support to fetal P-MSC growth. The immune properties of maternal P-MSCs were not analyzed in the present study.

Another property of clinical significance for P-MSCs is their differentiation potential. In the present study, P-MSCs cultured in hCBS were differentiated into mesenchymal and ectodermal lineages, indicative of multiple/pluri-potent characteristics. A recent study showed that P-MSCS were less prone to osteogenic differentiation and expressed a lower level of CD146 when compared with MB-MSCs (34), yet another study showed successful generation of an osteogenic graft from P-MSCs (35). It appears that the expression of phenotypes and functions by P-MSCs may vary when being cultured in different conditions or being isolated from different tissue origins. Indeed, we have observed that P-MSCs isolated from different donors may vary significantly in terms of proliferation capacities even when cultured under the same conditions (unpublished data). The question of why different observations have been made for P-MSCs has to be addressed under tightly controlled and well-defined conditions. 
FBS is a well proven tissue culture component for in vitro cell expansion, but its animal origin limits its use in clinic-compliant protocols. Our present work demonstrates that hCBS is an alternative to FBS for in vitro processing of human P-MSCs. Although the effective components in hCBS are yet to be defined, it is not difficult to hypothesize that P-MSCs can be well adapted to this in vitro condition considering that prior to being isolated the cells in the placenta were nourished mainly by CBS-borne nutrients and growth factors. This may, at least in part, explain why cells in homologous hCBS grew better than in non-homologous hCBS. Taken together, the results in this study suggest that homologous hCBS may support full stemness for human P-MSCs, and may hence be used as an alternative to FBS in clinically compliant procedures for processing human P-MSCs.

\section{Acknowledgements}

This study was supported in part by the National Natural Science Foundation of China (NSFC Program No. 30960176) (J.W.).

\section{References}

1. Brooke G, Cook M, Blair C, et al: Therapeutic applications of mesenchymal stromal cells. Semi Cell Dev Biol 18: 846-858, 2007.

2. Le Blanc K, Frassoni F, Ball L, et al: Mesenchymal stem cells for treatment of steroid-resistant, severe, acute graft-versus-host disease: a phase II study. Lancet 371: 1579-1586, 2008.

3. Sun LY, Kentaro A, Zhang HY, et al: Mesenchymal stem cell transplantation reverses multiorgan dysfunction in systemic lupus erythematosus mice and humans. Stem Cells 27: 1421-1432, 2009.

4. Centeno CJ, Busse D, Kisiday J, Keohan C, Freeman M and Karli D: Increased knee cartilage volume in degenerative joint disease using percutaneously implanted, autologous mesenchymal stem cells. Pain Physician 11: 343-353, 2008.

5. Sekiya I, Larson BL, Smith JR, Pochampally R, Cui JG and Prockop DJ: Expansion of human adult stem cells from bone marrow stroma: conditions that maximize the yields of early progenitors and evaluate their quality. Stem Cells 20: 530-541, 2002.

6. Parolini O, Alviano F, Bergwerf I, et al: Toward cell therapy using placenta-derived cells: disease mechanisms, cell biology, preclinical studies, and regulatory aspects at the round table. Stem Cells Dev 19: 143-155, 2010.

7. Chang CJ, Yen ML, Chen YC, et al: Placenta-derived multipotent cells exhibit immunosuppressive properties that are enhanced in the presence of interferon-gamma. Stem Cells 24: 2466-2477, 2006

8. Magatti M, De Munari S, Vertua E, Gibelli L, Wengler GS and Parolini O: Human amnion mesenchyme harbors cells with allogeneic T-cell suppression and stimulation capabilities. Stem Cells 26: 182-192, 2008.

9. Bailo M, Soncini M, Vertua E, et al: Engraftment potential of human amnion and chorion cells derived from term placenta. Transplantation 78: 1439-1448, 2004.

10. Li H, Niederkorn JY, Neelam S, et al: Immunosuppressive factors secreted by human amniotic epithelial cells. Invest Ophthalmo Vis Sci 46: 900-907, 2005.

11. Magatti M, De Munari S, Vertua E, et al: Amniotic mesenchymal tissue cells inhibit dendritic cell differentiation of peripheral blood and amnion resident monocytes. Cell Transplant 18: 899-914, 2009.

12. Pittenger MF, Mackay AM, Beck SC, et al: Multilineage potential of mesenchymal cells. Science 284: 143-147, 1999.

13. Mareschi K, Ferrero I, Rustichelli D, et al: Expansion of mesenchymal stem cells isolated from pediatric and adult donor bone marrow. J Cell Biochem 97: 744-754, 2006.
14. Evangelista M, Soncini M and Parolini O: Placenta-derived stem cells: new hope for cell therapy. Cytotechnology 58: 33-42, 2008.

15. Barlow S, Brooke G, Chatterjee K, et al: Comparison of human placenta- and bone marrow-derived multipotent mesenchymal stem cells. Stem Cells Dev 17: 1095-1107, 2008.

16. Portmann-Lanz CB, Schoeberlein A, Huber A, et al: Placental mesenchymal stem cells as potential autologous graft for preand perinatal neuroregeneration. Am J Obstet Gynecol 194: 664-673, 2006.

17. Sakuragawa N, Kakinuma K, Kikuchi A, et al: Human amnion mesenchyme cells express phenotypes of neuroglial progenitor cells. J Neurosci Res 78: 208-214, 2004.

18. Wei JP, Zhang TS, Kawa S, et al: Human amnion-isolated cells normalize blood glucose in streptozotocin-induced diabetic mice. Cell Transplant 12: 545-552, 2003.

19. Roelen DL, van der Mast BJ, in't Anker PS, et al: Differential immunomodulatory effects of fetal versus maternal multipotent stromal cells. Hum Immunol 70: 16-23, 2009.

20. Brooke G, Rossetti T, Pelekanos R, et al: Manufacturing of human placenta-derived mesenchymal stem cells for clinical trials. Br J Haematol 144: 571-579, 2008.

21. Mannello F and Tonti GA: No breakthroughs for human mesenchymal and embryonic stem cell culture: conditioned medium, feeder layer,or feeder-free; medium with fetal calf serum, human serum, or enriched plasma; serum-free, serum replacement nonconditioned medium, or ad hoc formula? all that glitters is not gold! Stem Cells 25: 1603-1609, 2007.

22. Tuschong L, Soenen SL, Blaese RM, Candotti F and Muul LM: Immune response to fetal calf serum by two adenosine deaminase-deficient patients after T cell gene therapy. Hum Gene Ther 13: 1605-1610, 2002.

23. Spees JL, Gregory CA, Singh H, et al: Internalized antigens must be removed to prepare hypoimmunogenic mesenchymal stem cells for cell and gene therapy. Mol Ther 9: 747-756, 2004.

24. Berger MG, Veyrat-Masson R, Rapatel C, Descamps S, Chassagne $\mathrm{J}$ and Boiret-Dupre $\mathrm{N}$ : Cell culture medium composition and translational adult bone marrow-derived stem cell research. Stem Cells 24: 2888-2990, 2006.

25. Lam AC, Li K, Zang ZB, et al: Preclinical ex vivo expansion of cord blood hematopoietic stem and progenitor cells: duration of culture; the media, serum supplements, and growth factors used; and engraftment in NOD/SCID mice. Transfusion 41: 1567-1576, 2001.

26. Kim YM, Jung MH, Song HY, et al: Ex vivo expansion of Human umbilical cord blood derived $\mathrm{T}$ lymphocytes with homologous cord blood plasma. Tohoku J Exp Med 205: 115-122, 2005.

27. Shetty P, Bharucha K and Tanavde V: Human umbilical cord blood serum can replace fetal bovine serum in the culture of mesenchymal stem cells. Cell Biol Int 31: 293-298, 2007.

28. Mizuno N, Shiba H, Ozeki Y, et al: Human autologous serum obtained using a completely closed bag system as a substitute for foetal calf serum in human mesenchymal stem cell cultures. Cell Biol Int 30: 521-524, 2006.

29. Xia SX, Gao YZ, Bian SZ, et al: Genetic polymorphisms of two STR loci D2S1399 and D5S2500 in eastern Chinese Han population. Fa Yi Xue Za Zhi 20: 200-201, 2004.

30. Nievas P, Martinez Jarreta B, Abecia E and Lareu MV: Fluorescence based amplification of the STR loci D18S535, D1S1656 and D12S391 in a population sample from Aragon (North Spain). Int J Legal Med 113: 58-59, 1999.

31. Parolini O, F Alviano, GP Bagnara, et al: Concise review: isolation and characterization of cells from human term placenta: outcome of the first international Workshop on Placenta Derived Stem Cells. Stem Cells 26: 300-311, 2008.

32. Caplan AL: Why are MSCs therapeutic? New data: new insight. J Pathol 217: 318-324, 2009.

33. Fazekasova H, Lechler R, Langford K and Lombardi G: Placenta-derived MSCs are partially immunogenic and less immunomodulatory than bone marrow-derived MSCs. J Tissue Eng Regen Med 5: 684-694, 2011.

34. Pilz GA, Ulrich C, Ruh M, et al: Human term placenta-derived mesenchymal stromal cells are less prone to osteogenic differentiation than bone marrow-derived mesenchymal stromal cells. Stem Cells Dev 20: 635-646, 2011.

35. Mohr S, Portmann-Lanz CB, Schoeberlein A, Sager R and Surbek DV: Generation of an osteogenic graft from human placenta and placenta-derived mesenchymal stem cells. Reprod Sci 17: 1006-1015, 2010 . 